\title{
Constructing a diversified ecological town system to promote economic restructuring and upgrading
}

\author{
Dan Liu ${ }^{1, \text { a, }}$, Xiaoyue Liu ${ }^{2, b}$ \\ ${ }^{1}$ Business School, Jianghan University, Wuhan, China \\ ${ }^{2}$ The University of Queensland Business School Business, Economics \& Law Postgraduate \\ a 136782581@QQ.com, bxiaoyue.liu.jenny@gmail.com, \\ *Corresponding author
}

Keywords: Ecological town, reverse urbanization, node economy

\begin{abstract}
The rise of the small town economy is the inevitable result of the reverse development of the city's gathering interests and congestion costs, as well as a new exploration to promote urban-rural integration and practice sustainable development. The construction of a diversified ecological town economic system will involve the reconfiguration of funds, people and land. In the reality of our country, this allocation of resources may face the situation of several institutional obstacles and trade-offs. Thus,the effiecient measures regarding constructing a diversified ecological town system for economic restruction and upgrade can be summerizsed as 1) understanding the impact of building a diversified ecological town economic system on China's economic structural transformation and economic development from a strategic perspective; 2) encouraging all regions to develop ecological towns according to local conditions; promoting the reform of the land system; 3) improving the liquidity of land resources within the scope of planning; 4)reducing the institutional costs in the development and construction of the ecological town economic system under the overall management policy of the country on cultivated land; 5) maintaining the synchronization of external environment transformation and ecological town construction.
\end{abstract}

\section{Introduction}

After the rapid development in the early stage, structural imbalance has become a new problem that restricts the continued development of China's economy. Among them, there is an imbalance in production structure that overcapacity in a number of traditional industries, and imbalances in ecological structure, which the gathering interests of big cities are beginning to be swallowed up by its crowding costs. Environmental pollution, traffic congestion, and high cost of living have become prominent problems facing many large cities in China. Therefore, exploring the modern style of the small town economy has become a new trend of reverse urbanization and supply-side reform.

\section{Small town economy and economic transformation}

Since the commercial civilization, the city has become the center of social and economic. And the development of the machine industry has strengthened this central position. Urban centralists believe that the aggregation effect makes the city the pillar of modern economic development. Although the increase in production and living costs will erode the benefits of aggregation as the city expands, the market economy can be adjusted through the industrial structure adjustment within the domain (e.g., moving out of traditional industries with more land occupation and serious pollution, and developing modern services with low- natural resource consumption and low-emission, as well as high-tech and smart industries, to ease the city's environmental pressure. However, changes in the real economy no longer support this assertion. On the one hand, the development of information Internet, high-speed transportation network, and Internet of Things has greatly reduced the interval transaction cost, which has weakened the value of urban aggregation 
effect. On the other hand, cities are mainly products of industrialization, and their functional value to machine industry may not be valuable for the high-tech, intelligent, and modern service industries in the era of knowledge and information. In the case of industrial characteristics and external conditions, there is no need for emerging industries to enter the city to bear the high pollution, congestion and land costs of which are produced by traditional industries. So we can see that when the Silicon Valley, the Greenwich town in the United States, and the Yunqi town in Hangzhou, China, emerged, the economic model based on the central city as the headquarters and small and medium-sized cities began to disintegrate. The development of a diversified small town economy built on the basis of characteristic industries has made people see the prospect of a new spatial model of modern economic.

The modern small town economy is a kind of activity space with residential towns, relying on unique resources and favorable ecology, living in a green way, symbolizing the characteristics industry, gathering the power of elements, forming a node-type economy with wide-area radiation and interconnection. Early ecological towns, relying on beautiful scenery or human heritage, have become places of sightseeing, such as the town of Davos in Switzerland and the town of Provence in France. But the modern economic towns are mostly industrial gatherings created by people who have fled the big cities, such as the Silicon Valley and Greenwich towns in the United States. The rise of the small town economy is the inevitable result of the reverse development of the city's gathering interests and congestion costs, as well as a new exploration to promote urban-rural integration and practice sustainable development. According to incomplete statistics, as of November 2015, less than one year after the implementation of the characteristic town construction, the first batch of 37 key towns in Zhejiang Province gathered more than 3,300 enterprises, attracting 13,000 technological talents, high-tech industry output value and tax revenue increased by an average of $20 \%$, the vitality of the town's economy has emerged.

Similar to the Internet system, the overwhelming, metropolitan-centered economy will be replaced by a distributed economy with small towns as nodes. This is a kind of low internal structure energy consumption (small scale, low consumption, low friction, environmental relaxation), efficient connection between nodes (small towns and towns, towns and cities) via high-speed information network and high-speed traffic network, diversified and efficient economic system. It has created a new spatial combination of labor professional division and is more conducive to the development of light assets and low energy industries such as information technology, research and development, intelligent industry and modern service industry. In the long term, this is in line with the direction of China's economic restructuring, and it has also expanded the use of resources on a broader and more uniform level. In the short term, the layout of the eco-small economic system itself will lead to a round of extensive, long-lasting and large-scale investment, including industrial innovation, real estate, the Internet and transportation facilities. This will undoubtedly change the current unfavorable situation of insufficient aggregate demand.

\section{The problems in constructing a diversified ecological town economic system}

As a reverse urbanization process, the construction of a diversified ecological town economic system will involve the reconfiguration of funds, people and land. In the reality of our country, this allocation of resources may face the situation of several institutional obstacles and trade-offs.

First of all, domestic and foreign experience has shown that the natural evolution of local economic ecology and the stimulation of external catalysts are the two main factors in the rise of the small town economy. Under the background of China's current system and the transformation of economic structure with top-level design, the government is an important external driving force for the economic construction and development of small towns. However, when the government tries to promote the development of ecological towns by external intervention, can it effectively prevent "agent impulses", especially the negative impact of movement-type construction of ecological towns, and fully coordinate the "two hands" respective roles will determine the actual effectiveness of building an ecological town. Theoretical and practical experience shows that playing the role of the government's strategy at the macro level and exerting the role of resource allocation in the 
market at the micro level will be an appropriate choice for obtaining positive results of active and actionable actions.

Second, the process of spreading economic loads to the periphery of the city through ecological towns will impact China's existing agricultural and rural land use systems in two ways. On the one hand, the government has established strict farmland management and contract management systems for the sake of safeguarding national economic security and stabilizing agriculture and farmers. The construction of the small town economic system will inevitably involve the occupation of new land. Although we can avoid the occupation of cultivated land through the transformation of old towns, the construction of large-scale high-speed transportation network supporting the small town economy cannot completely avoid the occupation of cultivated land. On the other hand, livable and suitable industry is an important condition for the sustainable development of ecological towns. When the urban population flows backwards to the countryside with the development of the town's economy, the current management system and regulations of rural housing and housing sites are limited. The way the original urban population buys houses from farmers in the form of market transactions. If the method of full leasing is adopted, the limited supply of houses in small-scale areas will easily lead to partial imbalance of supply and demand, which will lead to drastic fluctuations in house leasing prices and curb the survival and development of the town economy due to cost reasons. In fact, China's rural land policy currently plays a role in keeping farmers on the land, which is also an important basis for China's dual economic structure. The pressure on the land policy reform in the construction of a diversified ecological town economic system provides us with an opportunity to go to the dual structure and improve the liquidity and effective use of resources.

Third, as a node of the network economy, the vitality of the ecological town depends on the interconnection with the extraterritorial economic system. Therefore, with the growth of the small town economy, the development of related industries that undertake Unicom services is also an indispensable external support condition. This will involve the transformation of related industries, especially the logistics industry. In addition, the establishment of a decentralized social public service system that responds to the small town economy, such as medical care and education, will also be the system parameters involved in the construction of a diversified ecological town.

Fourth, in the modern economy, the leading role of capital flows to other resource flows is pioneering, and its activity also determines the effectiveness of the town's economic system. Meanwhile, speculative funds have traditionally been the most active factor in expansion. How to form a positive and reasonable income-risk sharing and combination mechanism will stimulate and test the wisdom of market participants and managers.

\section{Policy recommendations}

Based on the above analysis and assessment of relevant domestic and international experience, we propose the following policy recommendations for the construction of a diversified ecological town economic system in China's economic transformation.

From a strategic perspective, we will understand the impact of building a diversified ecological town economic system on China's economic structural transformation and economic development. Fully understand the evolutionary direction of the economic structure in the ecological evolution of interconnection. It will combine the economic system of building a diversified ecological town with the long-term development of the economy and the current cross-border. From the perspective of the top-level design, this systematic project is scientifically and rationally planned, and a development guideline is formulated. By publishing industry guidance catalogues and negative lists, we will provide clear expectations for market decision-making, and regulate the construction of a diversified ecological town economic system from the perspective of social management, and give play to the government's macro-strategic leading role.

Encourage all regions to develop ecological towns according to local conditions, and encourage them to carry out diversified selection and competition within the framework of the general outline to enhance the vitality and stability of the economic system. The vitality of the industry itself is the 
premise of the existence and development of the small town economic system. It is the most effective development path for entrepreneurs to choose and work hard under the market selection mechanism. To this end, it is necessary to reasonably define the respective roles and functions of local and grassroots government departments and development and construction enterprises, and formulate scientific and rational rules for the coordination of diversified participation rights and behaviors. Under the premise of reducing access restrictions, attract social investment through fair competition mechanisms and preferential packages (land and related real estate rents - taxation social and economic contributions - environmental contributions, etc.) and allow them to be in the aforementioned framework and norms (Self-determination, input and innovation in social rationality).

Promote the reform of the land system, improve the liquidity of land resources within the scope of planning, and reduce the institutional costs in the development and construction of the ecological town economic system under the overall management policy of the country on cultivated land. Change the current single arable land area index scheme in cultivated land management to establish a more reasonable "effective capacity system", and make non-urban land more effective use under strict supervision. At the same time, design an effective mechanism for the housing estates in the planning area to enter the market, allowing farmers to choose their own rights and disposal and lifestyle.

Design and establish an effective ecological town governance mechanism to play a fundamental role in ensuring full freedom of exploration and innovation, maintaining the symbiotic benefits of town building participants and various resource elements, and promoting and ensuring efficient resource flows. Constructing an ecological town in an open mode, through the exploration and construction of the operation model, and shaping the corresponding practices and cultures, the town becomes a gathering city, a learning place, a river of communication, and a boat for leadership.

Maintain the synchronization of external environment transformation and ecological town construction, and establish a distributed public service system based on the original public service resources of the central city. Encourage and support innovative companies to develop more efficient and low-cost logistics and distribution technologies and operation models to fully enhance the operational efficiency of the new network economic system. In the construction of the relevant high-speed transportation network, the implementation of the elevated and less lane construction scheme will be implemented to minimize the impact on cultivated land. Reduce construction costs through scientific planning and technological advancement.

Improve the liquidity of funds flow and the environment of the investment and financing. Conduct targeted institutional design for investment and financing of ecological town economic system. Demonstrate and construct a special financial platform to reinforce standards (including bank funds, venture capital, securitization investment, and speculative funds related to Industrial development, technological innovation, land development), which plays a supportive role in positive connectivity and risk allocation..

Conduct comprehensive exploration and exploration of all aspects and ecological elements in the construction of ecological towns in advance to avoid the risk of blind investment and establish demonstration models for subsequent large-scale development.

\section{References}

[1] Sheng shihao, Zhang Weiming. ( 2016 ) Special Town: A Form of Industrial Spatial Organization .Zhe Jiang Social Sciences, 03, 36-38.

[2] Cao Zongping. (2016)Internal Motivation, External Conditions and “Counter Urbanization”. Reform, 01, 88-94.

[3] Chen Bin. (2015)Study on the Problem of "Inverse Urbanization" in the Process of New Urbanization. Macroeconomic Management, 7, 31-33.

[4] KeShanzi and Zhao Yao. (2014)Industrial Structure, City Size and Urban Productivity in China. 
Economic Research Journal,04,76-88.

[6] Zhang Peng. (2015) Discrimination and Transcendence of the S Curve of Urbanization Development from the Perspective of Central Land Theory. Journal of Commercial Economics, 7, 47-49. 